\title{
Quantitative Correlative Light and Electron Microscopies; Targeting the Host Actin Cytoskeleton
}

\author{
Niels Volkmann and Dorit Hanein
}

Bioinformatics and Structural Biology Program, Sanford Burnham Medical Research Institute, La Jolla, USA

Recent advances in high-resolution light microscopies, the new generation of electron cryomicroscopes, new state-of-the-art direct electron detectors available today, together with the emerging correlative light and electron microscopy techniques provide the means to tie dynamic biological processes with the underlying structural correlates in situ. The major challenge is to quantitatively link macroscopic cellular outputs to molecular-resolution structural changes in the crowded cellular environment. Molecular understanding of all system components becomes truly meaningful only if it can be related to the components working as a three-dimensional ensemble within a living cell. In this presentation, we will provide snapshots of our current advances targeting quantitative correlation of the dynamic state of a biological pathway with high-resolution structural information in the same window of time and space, using cell-matrix adhesions as model systems.

A reductionist approach in studying functional-structural correlates of mammalian cell actin cytoskeleton provides details primarily at the level of individual proteins and binding partners. Much less is known about the interactions between these proteins in situ, how they are regulated and how they determine signaling outputs. The major challenge is to establish approaches that can robustly capture these assemblies at high-resolution structural detail and correlate them with defined functional states in a cellular environment. To quantitatively correlate over extended length scales and orders of magnitude in time requires multidisciplinary, hybrid experimental approach. In this presentation, we will describe our advances in seamlessly tying experimental and computational tools to allow structural integration of molecular assemblies at cellular levels. To that end, we will aim at describing our recent improvement in cell culture protocols, high-resolution imaging modalities, biophysical assays in conjunction with algorithms and automation approaches for data processing and data mining to achieve optimally combined workflows [1].

Here, using this correlative approach [2], we will provide evidence that Toxofilin, an actin-binding protein secreted by invading Toxoplasma gondii parasites into the host cell cytoplasm at the onset of Toxoplasma invasion, targets the host cortical actin cytoskeleton to facilitate invasion [3]. This protozoan parasite belongs to the phylum apicomplexa, which also includes Plasmodium, the agent of malaria. T. gondii quickly establishes infection as a fast-replicating form, the tachyzoite, which invades numerous cell types throughout the body. The parasite can cause encephalitis and neurological diseases and can also negatively affect the heart, liver, and eyes (chorioretinitis).

Correlative light and electron microscopy combined with electron tomography followed by $3 \mathrm{D}$ analysis, allowed tracking toxofilin, an actin binding protein, in action within the host mammalian cell and showed that integrity of the host cortical actin cytoskeleton was disrupted at the site of parasite entry, suggesting that toxofilin induces local actin de-polymerization. Quantitative fluorescent speckle microscopy to measure actin filament dynamics, indicates that toxofilin facilitates invasion by upregulating host cortical actin filament turnover, consistent with its monomer sequestration and barbed 
end filament capping capabilities in vitro. The limited amount of protein restricted to the site of entry is fully compatible with an actin disassembling or severing activity working at sub-stoichiometric ratios with actin. The ability of toxofilin to expedite invasion by regulating the turnover of cortical actin filaments in the host cell is likely to confer a selective advantage to the parasite.

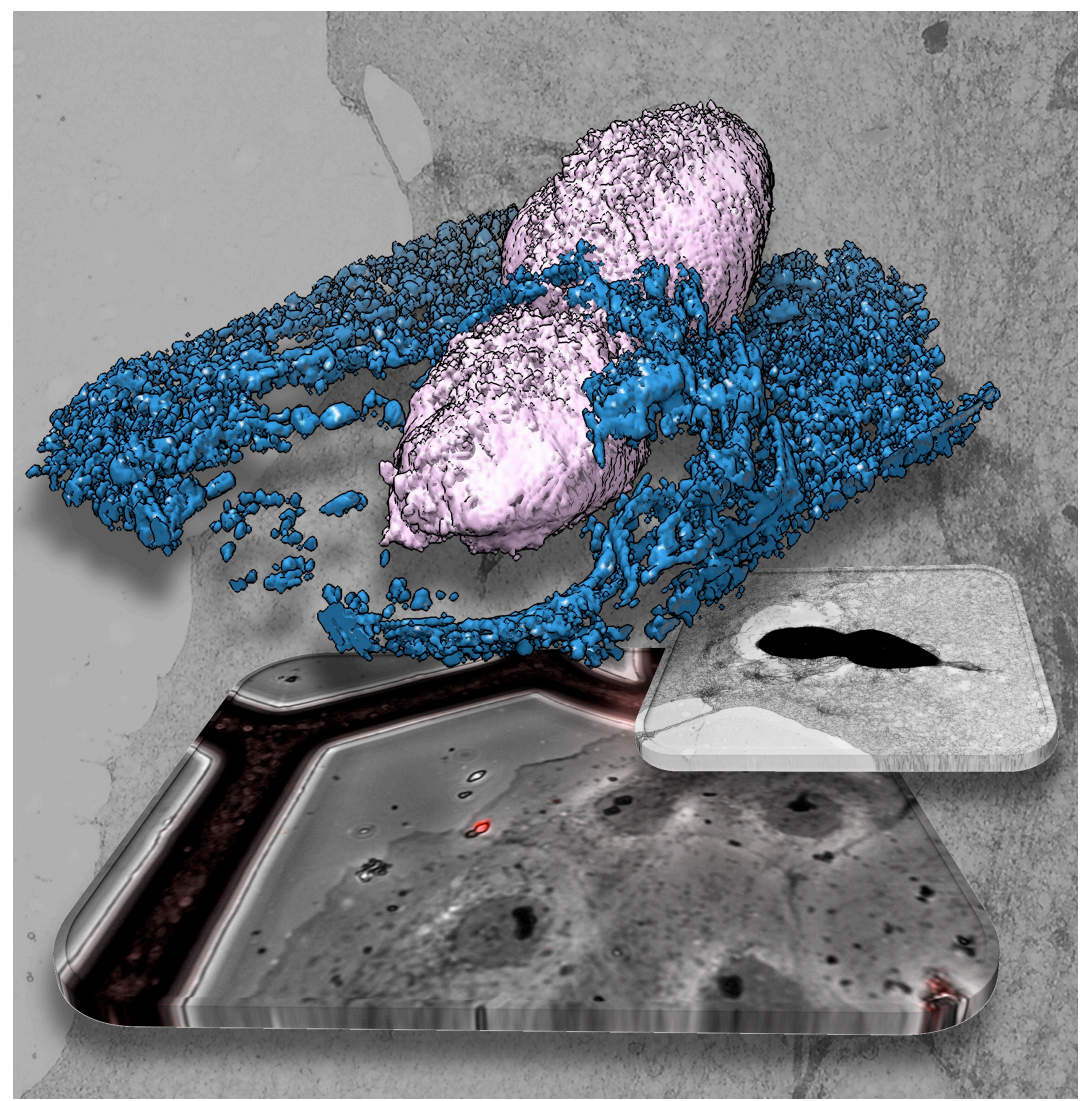

Figure 1: Detection of an invading Toxoplasma tachyzoite (pink) and of the host cell actin meshwork (blue) at the site of entry, by applying correlative light and electron microcopy as well as electron tomography and three-dimensional (3D) image analysis. Toxofilin secretion by the invading parasite is associated with local disassembly of the host cell cortical actin meshwork. Images designed by Karen L Anderson, Violaine Delorme, Dorit Hanein and Niels Volkmann [3]

[1] These studies are supported by the National Institute of General Sciences (NIGMS) Grant Number P01 GM098412 (DH and NV).

[2] Hanein D, Volkmann N. Correlative light-electron microscopy. Adv Protein Chem Struct Biol. $\underline{82}$, 91-99. (2011).

[3] Delorme-Walker V, Abrivard M, Lagal V, Anderson K, Perazzi A, Gonzalez V, Page C, Chauvet J, Ochoa W, Volkmann N, Hanein D, and Tardieux I. Toxofilin upregulates the host cortical actin cytoskeleton dynamics, facilitating Toxoplasma invasion. J Cell Sci. $\underline{125}$, 4333-4342. (2012).

[4] Lee, S. H., Hayes, D. B., Rebowski, G., Tardieux, I. and Dominguez, R. Toxofilin from Toxoplasma gondii forms a ternary complex with an antiparallel actin dimer. Proc. Natl. Acad. Sci. USA 104, 16122-16127. (2007). 